\title{
Uncovering Community Structure in Geospatial Networks: A Case Study of Urban Bus Networks in Hangzhou, China
}

\author{
Yujing Wang a,b, Fu Ren ${ }^{a, b}$, Qingyun Du ${ }^{\text {a, }}$ * \\ ${ }^{a}$ School of Resources and Environmental Science, Wuhan University, afreeboat@whu.edu.cn; renfu@whu.edu.cn;qydu@whu.edu.cn \\ ${ }^{b}$ Key laboratory of Geographic Information Systems, Ministry of Education, Wuhan University, afreeboat@whu.edu.cn; \\ renfu@whu.edu.cn;qydu@whu.edu.cn \\ * Corresponding author
}

Keywords: Geospatial Network, Complex Network, Disjoint Community, Overlapping Community, Spatial Resistance, Spatial Contiguity

\begin{abstract}
:
Complex systems are very often organized under the form of networks, where nodes and edges are embedded in the geographic space in which human beings live. A geospatial network is a complex network that includes road networks, flight connections, physical internet infrastructures, and power grids, etc. The properties of geographic spaces and network topologies are key to the geospatial network function and evolution. It is crucial to characterize and understand the structure and evolution of these geospatial networks for different domains, ranging from social sciences to geographic sciences. Community structures, whose nodes often cluster into tightly knit groups (with a high density of within-group edges and a low density of between-group edges), have been given much attention because of their significance in analyzing complex geospatial networks. Community structures, which account for the functionality of a complex system in geographic space, represent a partition (disjoint community) or cover (overlapping community) of a geospatial network that is expected to have an optimal solution. Community structure found in geospatial network, such as those present in networks capturing the movements of people or animals, are subject to environmental factors, physical constraints, and other spatial influences. Therefore, communities in spatial networks often have geo-graphic meanings such as neighborhood boundaries, urban structures, and habitat territories. Moreover, the influence of geospatial indicators on the detection of community structure must not be overlooked.
\end{abstract}

Compared with disjoint community structures, overlapping community structures are more ubiquitous community structures, which represent the fundamental characteristic of any complex network form in the physical world. In a geospatial network, community structures represent the different functions or structural units of a geographic space. Furthermore, overlapping communities are the result of the network evolution process, which represent the share channel of a neighbouring community. In geographical space, overlapping communities are always in the middle of two different communities. Spatially and functionally, overlapping communities are the bridge in communication between neighbouring communities. Therefore, the detection of overlapping communities in geospatial networks is of great significance. Although there are many methods for the detection of overlapping community structures, existing algorithms usually focus on network topology characteristics and ignore the effects of geospatial attributes on overlapping community structures.

Hence, in this paper, we proposed a new methodology for uncovering communities in geospatial networks. The focus of our methodology is the spatially constrained speaker-listener label propagation algorithm (SLPA), which adds spatial resistance and spatial adjacency relations to the community detection algorithm. Furthermore, we make a case study using urban bus network in Hangzhou, China. The results show that the algorithm is efficient and reasonable to discovering overlapping community structures in geospatial networks. Overall, this study will help to understand and monitor the spatial interaction development of geographic phenomena and explore the functional characteristics of the urban complex system that are useful and meaningful for policy makers and planners. 